\title{
Effects of sulfhydryl binding reagents on the photoresponses of
} amphibian retinal rods

\section{Donner, K.}

Elsevier

1989

Comparative Biochemistry and Physiology. 1989. 94: 125-132.

http://hdl.handle.net/1975/941

http://dx.doi.org/doi:10.1016/0300-9629(89)90795-0

Downloaded from Helda, University of Helsinki institutional repository.

This is an electronic reprint of the original article.

This reprint may differ from the original in pagination and typographic detail.

Please cite the original version. 


\title{
EFFECTS OF SULFHYDRYL BINDING REAGENTS ON THE PHOTORESPONSES OF AMPHIBIAN RETINAL RODS
}

\author{
Kristian Donner, ${ }^{*}$ Simo Hemilä and Ari Koskelainen \\ Laboratory of Physics, Helsinki University of Technology, SF-02150 Espoo, Finland; and \\ *Department of Zoology, University of Helsinki, SF-00100 Helsinki, Finland
}

(Received 20 February 1989)

\begin{abstract}
We have studied the effects of four sulfhydryl binding reagents (NEM, PHMB, PCMP and IAA) and the disulfide reducing agent, DTT, on the photocurrent of vertebrate rods by recording the ERG across the aspartate-treated retina of the frog, and by suction pipette recording from isolated rods of the tiger salamander.

2. SH-reagents brought about three types of effects on rods: (1) a fast transitory increase in light sensitivity and photocurrent; (2) a "leakage" current that could not be turned off by light; and (3) a slower irreversible loss of sensitivity.

3. The fast effects, including the leakage current, are attributable in part to direct action on the sodium channels in the plasma membrane.

4. NEM, PHMB and PCMP were able to affect the transduction machinery inside the rod, which first contributed to the growth of photoresponses, but gradually depressed light sensitivity irreversibly.

5. Typically, the reagents also induced large ERG transients of non-receptor (glial) origin.

6. The fast effects of DTT on isolated rods were similar to those of the SH-reagents. This drugi however, had no clear effect on ERG photoresponses, suggesting that the oxidation of sodium-channel SH-groups is modest in the intact retina as compared with isolated rods.
\end{abstract}

\section{INTRODUCTION}

The oxidation of sulfhydryl (SH) groups is implicated as a major factor in the ageing of the membranes of the rod outer segment (ROS), necessitating their comparatively rapid turnover (cf. Derevyanchenko et al., 1985; Ostrovskii et al., 1987). In osmotic studies on isolated ROSs, oxidizing conditions have been shown to abolish the light-sensitive permeability, while reducing conditions tend to preserve it (Bownds and Brodie, 1975; Wormington and Cone, 1978). Cone et al. (1984) found that the SH-binding reagents, NEM, PCMP, PMPS and IAA, as well as the disulfide reducing agent DTT, were able to reverse the effects of oxidation (restore osmotically measured sodium permeability), but that they also restored permeability when it had been abolished by light. These findings suggest a connection between the functioning of the sodium channel, its redox state, and the state of its SH-groups.

We have previously shown that mass rod photoresponses from the isolated aspartate-treated frog retina are transiently desensitized by oxidizing conditions, and sensitized by reducing conditions (Donner et al., 1987). The effects, however, were comparatively weak and involved no clear change in total light-sensitive current. The rods in the intact retina would thus appear to be reasonably well protected against harmful effects of oxygen.

In the present work we supplement the redox study with experiments on the effects of reagents that bind to SH-groups and/or bar the formation of disulfide bridges. The experiments were done both on intact isolated retinas of the frog and on single rods of the tiger salamander. The SH-reagents were found to produce an increased dark current which was only partly light-sensitive. We interpret these effects as changes in the channel molecule due to binding of the drugs to its SH-groups. Three of the SH-reagents (NEM, PHMB and PCMP) subsequently set off a continuous decrease in sensitivity, which we ascribe to action inside the rod. DTT, which serves to break disulfide bridges, also effectively increased the lightsensitive conductance in the isolated rod, but not in the intact retina. This agrees with our earlier conclusion that rods in situ are comparatively wellshielded against oxidation (i.e. the critical SH-groups are predominantly in the reduced state).

\section{MATERIALS AND METHODS}

Rod current studied by mass receptor potential and single-rod recording

The ERG mass receptor potential across the aspartatetreated retina offers some advantages over current recordings from single rods. The preparation is very stable, allowing long and reliable recording from the same retina, the signal/noise ratio is high, and each photoresponse in itself reflects the average of a large number of individual rod current responses. Also, the rods remain in a comparatively natural environment, embedded in the retina. The main drawback is that although synaptic transmission is blocked with aspartate, the ERG is contaminated by radial currents of other than receptor origin. Although these components can be largely revealed by suitable experimental procedure (see Donner and Hemilä, 1985; Donner et al., 1988), they do present an insurmountable obstacle to establishing the source at least of processes not sensitive to light, as associated with, e.g. the drugs used here. In the present work, we therefore chose to do the main bulk of experiments as ERG recordings on the isolated retina, and to check the conclu- 
sions in a smaller number of current recordings from single rods.

\section{Biological material and preparation}

The ERG experiments were done on the isolated, aspartate-treated retina of the frog, Rana temporaria. The frogs were caught in the autumn in southern Finland and stored in basins without feeding at $4^{\circ} \mathrm{C}$. The night before an experiment a frog was gradually warmed to $\mathrm{ca} 17^{\circ} \mathrm{C}$ in darkness. The frog was killed and double-pithed, both eyes were excised and the retinas were isolated under dim red light. One of the retinas was mounted in the specimen holder (see below) and left to dark-adapt for at least $1 \mathrm{hr}$ before the experiment started.

The current recordings were performed on single rods of the adult tiger salamander, Ambystoma tigrinum. The reason for using a different species was that the frog retina proved unexpectedly difficult to work with, giving a very low yield of light-sensitive isolated rods. It was thought that rods from another amphibian would not be decisively different as regards basic biochemistry. Larval tiger salamanders obtained from Lowrance Waterdog Farm Ltd. (Tulsa, OK, USA) were fed and metamorphosized at room temperature. Before an experiment, the animal was kept for at least $12 \mathrm{hr}$ in darkness. The dissection was carried out under dim red light and the isolated retinas were transferred to a watch glass containing $\mathrm{ca} 1 \mathrm{ml}$ Ringer kept in a dark container at ca $16^{\circ} \mathrm{C}$. The proportion of viable rods obtained clearly rose if the retinas were left to adjust to the new medium for some $4 \mathrm{hr}$ before the experiment. By gently tearing the retina under infrared light, isolated cells were obtained, of which about $90 \%$ were outer segments, but about $10 \%$ outer + inner segments used for the recordings. A small portion of the rod suspension was drawn into a syringe from the bottom of the watch glass and transferred to the experiment chamber.

\section{Recording and perfusion}

The mass receptor potential. The experimental set-up was as previously described (Donner and Hemilä, 1985; Donner et al., 1987, 1988). Briefly, the retina was placed receptors upwards in a specimen holder where the transretinal voltage was recorded with $\mathrm{Ag} / \mathrm{AgCl}$ electrodes. The receptor side of the retina was perfused with Ringer at $12^{\circ} \mathrm{C}$. The signal was continuously monitored on a storage oscilloscope and recorded on a slow pen recorder and, in addition, the photoresponses were recorded on a fast chart recorder. The ERG figures have been retraced by hand from these records.

The current of isolated rods. The current recording system was similar to that described by Baylor et al. (1979), except that our suction system was not closed. An open-ended oil-container was connected by a side-tube (inner diameter $0.64 \mathrm{~mm}$, length $60 \mathrm{~cm}$ ) to the tube connecting the micrometer-driven syringes to the pipette. Thus, pressures applied by the syringes were transient with a time-constant of about $10 \mathrm{sec}$. Before suction, the standing pressure difference between pipette and bath was adjusted close to zero (within a few pascals) by raising or lowering the open branch from outside the light-tight box while observing the movements of tiny particles in the suspension. A suitable rod was selected through an inverted microscope with video display under infra-red illumination. The inner segment was drawn into a silanized glass pipette $(10-11 \mu \mathrm{m}$ inner tip diameter) from the bottom of the specimen chamber. The bottom was covered with poly-HEMA [19,206-6 poly(2hydroxy-ethyl methacrylate); Folkman and Moscona, 1978], which appeared to prevent cells from adhering to the bottom more effectively than silane. After passing through a current-to-voltage converter and conventional amplification (high-pass cut-off at $130 \mathrm{~Hz}$ ), the signal was stored on diskette (digitization at 40 samples per second). It was also displayed on a storage oscilloscope and recorded on a slow pen recorder. The recordings were made at $c a .20^{\circ} \mathrm{C}$.
Stimulation. Stimuli were $0.1 \mathrm{sec}$ flashes of $493 \mathrm{~nm}$ light. The absolute intensity of the stimulating light was calibrated by spectrophotometrical measurement of the rate of rhodopsin bleaching in the intact frog retina.

Perfusion. The standard Ringer solution perfusing the receptor side of the retina in the ERG experiments contained $95 \mathrm{mM} \mathrm{NaCl}, \quad 3 \mathrm{mM} \mathrm{KCl}, \quad 0.9 \mathrm{mM} \mathrm{CaCl}_{2}$, $0.5 \mathrm{mM} \mathrm{MgCl}_{2}, 12 \mathrm{mM}$ phosphate buffer (pH 7.5), $10 \mathrm{mM}$ glucose and $2 \mathrm{mM}$ aspartate. In the experiments on isolated rods, the phosphate buffer was replaced by $8 \mathrm{mM}$ phosphate plus $4 \mathrm{mM}$ bicarbonate (which gave a better yield of photosensitive rods), and no aspartate was used. The gravitationcontrolled perfusion system involved two tubes joining at the inlet to the specimen chamber. The flow could be switched from standard Ringer to drug-containing Ringer by valves outside the light-tight box.

Four different sulfhydryl-binding reagents were used: $N$-ethylmaleimide (NEM), iodoacetamide (IAA), and two mercury-containing drugs: $p$-hydroxymercuribenzoate (PHMB) and $p$-chloromercuriphenylsulfonic acid (PCMP). The fifth drug used was the disulfide reducing agent dithiothreitol (DTT; all reagents by Sigma).

Determination of junction currents. When the solution bathing the outer segment is changed (e.g. by the introduction of a drug), the record could, in principle, be distorted by the resulting junction current. We therefore carefully measured the junction currents associated with the introduction of $2 \mathrm{mM}$ NEM and of $0.2 \mathrm{mM}$ IBMX. A non-responding rod outer segment was drawn into the recording pipette and changes in the baseline were recorded as the drug was introduced and washed out many times in succession. The junction current was smaller than $3 \mathrm{pA}$ with these drugs. Since such a small current is insignificant for our conclusions, we have not subtracted it from the records shown in the Figures.

\section{Analysis}

The response amplitude is here denoted $U$. In the ERG experiments, $U$ is a transretinal voltage change, and in the single-rod experiments, it is a change in the outer segment current. We distinguish between effects on the saturated response amplitude, $U_{\max }$ (whose dark-adapted pre-drug value is denoted $U_{0}$ ) and effects on the relative sensitivity to $\operatorname{dim}$ stimuli, $S=\left(U / U_{\max }\right) / I$, where $U$ is the amplitude of the response to a low stimulus intensity, $I$. (Observe that the absolute sensitivity, $U / I$, is then the product, $S U_{\max }$.) For better accuracy, the "dim" stimuli used were such that they gave response amplitudes $c a 10-30 \%$ of $U_{\max }$, i.e. outside the range of strictly linear $U-I$-dependence (cf. Baylor et al., 1974; Hemilä, 1977, 1987). Extrapolation to the linear range was achieved by fitting the response amplitudes with ad hoc stimulus-response curves, so called $z$-functions (one type of generalized Michaelis function; see Bäckström and Hemilä, 1979). Furthermore, in the ERG recordings, we preferred to use nearly ( $\mathrm{ca} 80 \%$ ) saturating rather than fully saturating flashes in order to avoid excessive glial responses (cf. Donner and Hemilä, 1985).

\section{RESULTS}

\section{The effects of NEM}

We first characterize in detail the effects of one of the sulfhydryl reagents, NEM, and then consider similarities and differences in the action of the other drugs.

Fast effects. A switch from standard Ringer to Ringer containing $2 \mathrm{mM}$ NEM induced two types of effects in the intact retina: (1) a fast voltage transient of polarity opposite to photoresponses, accompanied by larger photoresponses and enhanced light sensitivity; (2) a slower, irreversible decay of sensitivity. 


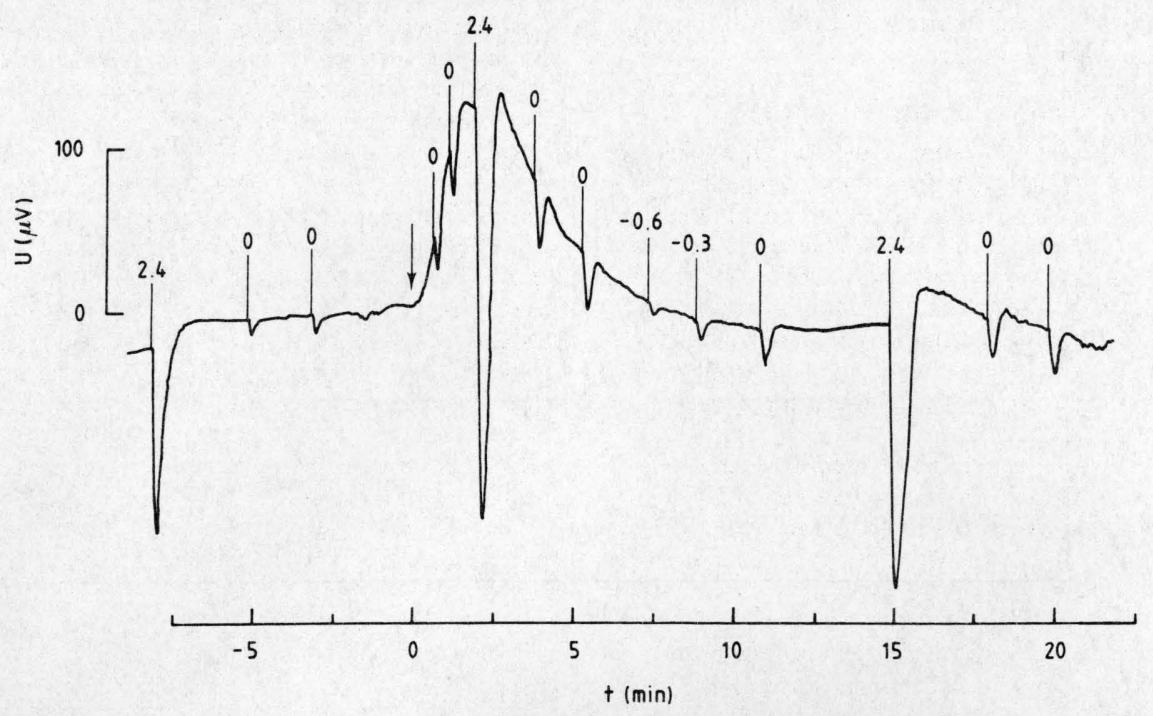

Fig. 1. Transretinal ERG voltage transient caused by a 1 min pulse of Ringer containing $2 \mathrm{mM}$ NEM. The arrow gives the time when the peak of the drug concentration reaches the retina. The numbers above the photoresponses give $\log _{10}$ relative flash intensities; $\log I=0$ corresponds to 2.5 photoisomerizations per rod. The responses to log intensity 2.4 are practically saturated.

To minimize the irreversible desensitization, which we thought could be due to the accumulation of NEM inside the ROS (see below), we used short pulses of NEM Ringer ( $10 \mathrm{sec}$ to $2 \mathrm{~min}$ ), rather than continuous perfusion in many of the ERG experiments on the frog retina.

Figure 1 shows an ERG record from the aspartatetreated frog retina during such a pulse experiment. The positive NEM-induced transient is seen to peak ca 2 min after the drug reaches the receptor surface of the retina. Its amplitude, denoted $U_{\mathrm{D}}$, in different experiments, was roughly proportional to the NEM dose (concentration $\times$ pulse duration), whereby $2 \mathrm{mM}$ NEM applied for $1 \mathrm{~min}$ gave an amplitude about equal to that of the pre-drug maximum light response $U_{0}$. At the peak, the amplitude of the saturating response $U_{\max }$ was increased by approximately the amount $U_{\mathrm{D}}$ (see Fig. 1), indicating that the transient is mainly accounted for by an increase in light-sensitive rod current.

Figure 1 also shows that, initially, the responses to weak stimuli grew relatively more than the saturated responses. This means that the relative sensitivity, $S$ (see Materials and Methods), increased. In other words, the stimulus-response curve in a $\log -\log$ plot moved upwards (log $U_{\max }$ increased) and to the left ( $\log S$ increased).

Experiments with NEM in continuous perfusion gave early effects (growth of sensitivity and dark current) that were basically similar to those seen in pulse experiments, but with one significant difference. This is illustrated by Fig. 2A. It is here evident that the initial transient (which peaks later than in the pulse experiments) cannot be entirely accounted for by the increase in the light-sensitive rod current. At the peak of the transient, the saturating responses failed to reach the previous saturation level (given by the left-most response in the Figure), indicating a residual component not sensitive to light. However, from the transretinal potential it is impossible to infer in what retinal cells this residual component originates.

Figure 2B shows the effects of continuous NEM perfusion on an isolated salamander rod, to be compared with the ERG record in Fig. 2A. Here, the presence of a light-insensitive component in the rod current itself is unequivocally established. Even at the early stage of the transient, saturating flashes fail to turn off all the current: the peaks of saturated responses remain $c a 10 \mathrm{pA}$ below the pre-drug saturation level. In other respects, the picture obtained from the ERG is corroborated.

Slow effects. In Fig. 2 it is also possible to see some of the fairly dramatic slow effects of NEM in continuous perfusion. The positive transient was followed by a steep reduction of sensitivity $S$ (in the ERG from ca $15 \mathrm{~min}$ onwards; in the isolated rod, much faster). The saturating response $U_{\max }$ also decreased, aithough less rapidly. In Fig. 3 , the changes in $\log S$ and $\log U_{\max }$ (relative to their pre-drug values) are plotted from the same ERG experiment of which a portion is shown in Fig. 2A.

After the initial rise to a maximum, a monotonical decay set in, which for $\log S$ is seen to be almost linear with time, i.e. sensitivity fell exponentially. The time constant was inversely related to the NEM concentration; with $0.5 \mathrm{mM}$ NEM (as in Fig. 3), it was about $4 \mathrm{~min}$ (range $3.5-5 \mathrm{~min}$ in five experiments). The saturating response amplitude decayed much less than sensitivity. In Fig. 3, $U_{\max }$ decreased only by ca $0.5 \log$ units over half an hour, while sensitivity fell more than $3 \log$ units.

In the isolated rod, diffusion distances and barriers are slight and sensitivity fell much faster. In Fig. 2B, stimulus intensity 0 is saturating in the pre-drug situation, whilst during the later phase of the drug transient, a $1.5 \log$ units stronger stimulus is seen to elicit responses of rapidly decreasing amplitude. This 

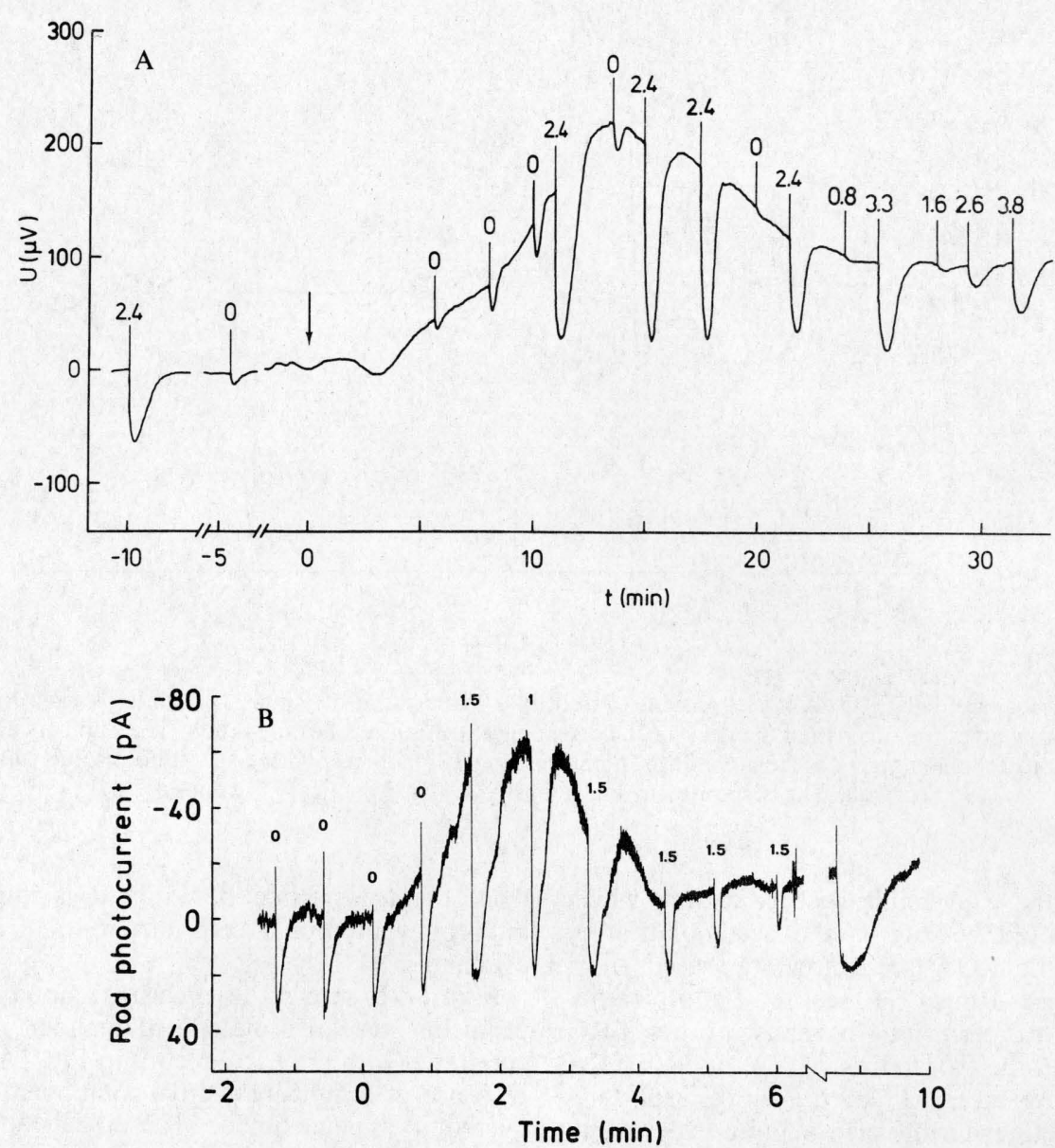

Fig. 2. (A) Transretinal ERG voltage transient after a switch from standard Ringer to continuous perfusion with Ringer containing $0.5 \mathrm{mM}$ NEM. The numbers above the photoresponses give log relative flash intensities as in Fig. 1. On the left are seen a response to a weak flash and a nearly saturated response before application of NEM. Note that after the positive transient the rapid decrease of sensitivity makes it necessary to use successively stronger intensities both for "dim" and for saturating flashes. (B) The current of an isolated rod before and after a switch from standard Ringer to continuous perfusion with Ringer containing $0.5 \mathrm{mM}$ NEM. All flashes were nearly or fully saturating, except the last two (after the peak of the transient), where the very steep fall in sensitivity made the intensity used $(\log I=1.5)$ quite inadequate. Far right the approximate saturation level as revealed by a $15 \mathrm{sec}$ square-wave of strong white light $(\log I>2.5)$. The $\log I$ values are relative and not directly comparable to those in (A).

is due to a decrease in relative sensitivity, $S$ : the saturating response at the right edge of the record shows that $U_{\max }$ at that stage was still even larger than before the introduction of NEM.

The waveform of responses. The waveforms of photoresponses during the later phases provide some further clues to the mode of action of NEM. Although the decrease in relative sensitivity, $S$, formally resembles light-adaptation in the sense of a rightward movement of the $\log$ intensity-response $(R-\log I)$ curve, responses to both weak and strong stimuli became considerably slower and more extended in time. Also, the $R-\log I$ curve tended to steepen, and facilitation by one flash of the response to a subsequent flash was observed. All these changes have been reported to occur in connection with strong inhibition of phosphodiesterase (Capovilla et al., 1983) and thus suggest one target for the internal action of NEM.

After long NEM perfusion, when sensitivity had decreased by $2-3 \log$ units and $U_{\max }$ fallen to a small fraction of $U_{0}$, quite peculiar photoresponses were often observed in the frog ERG (see Fig. 4). In these responses, a sharp positive peak preceded the photoresponse proper. Such responses have been seen under various conditions where the tip of the ROS is desensitized more strongly than the base (cf. Donner et al., 1987). A detailed consideration will be deferred to the Discussion.

\section{The effects of other $\mathrm{SH}$-reagents}

After extensive studies of the action of NEM, the basic experiments were repeated with three other sulfhydryl reagents: PHMB, PCMP and IAA. 


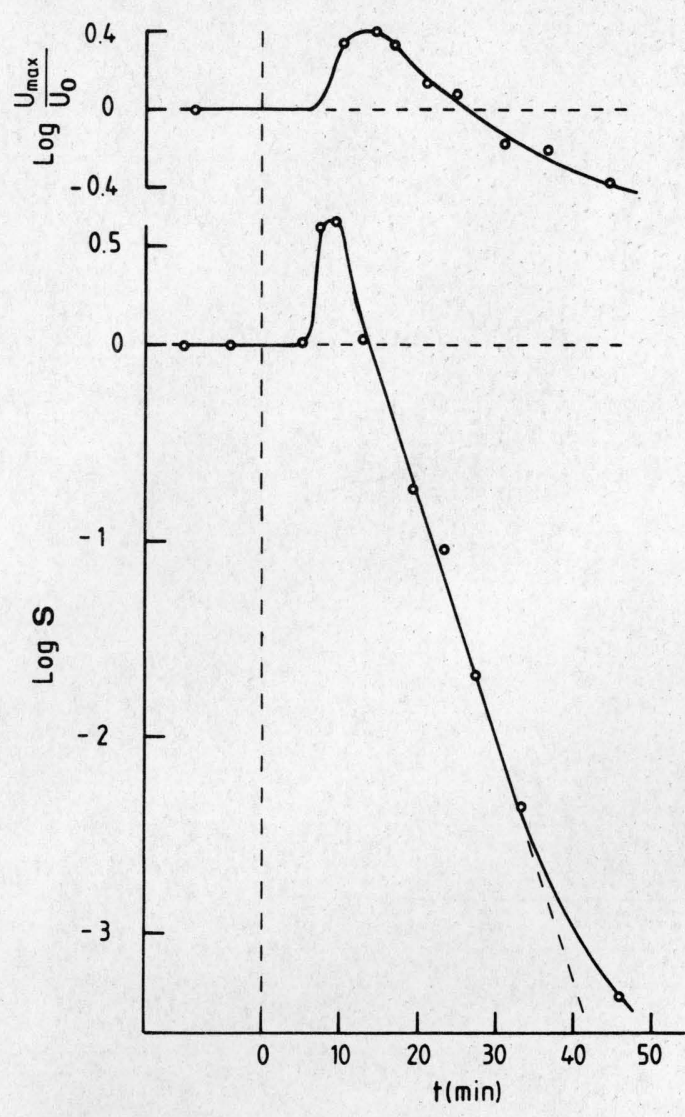

Fig. 3. Changes in $\log S$ and $\log U_{\max }$ extracted from the experiment shown in Fig. $2 \mathrm{~A}$ (continuous perfusion with $0.5 \mathrm{mM}$ NEM). $\log S=0$ corresponds to $S^{-1}=11$ photoisomerizations per rod.

In the frog ERG, both of the mercury-containing drugs (PHMB and PCMP) caused very large positive transients, up to $U_{\mathrm{D}}>10 U_{0}$. However, as seen in the PHMB experiment of Fig. 5A, the saturating response amplitude $U_{\max }$ did not grow much during these transients. The largest increase of $U_{\max }$ observed after a $0.5 \mathrm{~min}$ pulse of $0.6 \mathrm{mM}$ PHMB was $0.7 U_{0}$; and indeed, comparison with the single-rod record in Fig. 5B shows that the large drug-induced transient has little to do with rod current, but is probably mainly of glial origin. In Fig. 5B there is a moderate initial increase in dark current with a roughly corresponding growth of photoresponses, followed by a gradual inactivation of phototransduction. As with NEM, later photoresponses got more extended than in standard Ringer.

PCMP induced the largest light-insensitive "glial" ERG transients of all the SH-reagents. In the singlerod recordings, however, the picture was again very similar to that seen with NEM and PHMB, involving a transient increase in dark current and $U_{\max }$ followed by a decay phase with slowed response kinetics.

The fourth SH-reagent, IAA, also boosted saturating responses, but less so that NEM. In the frog ERG, a $1 \mathrm{~min}$ pulse of $2 \mathrm{mM}$ IAA approximately doubled $U_{\max }$. In addition, the initial ERG transient contained a largish "glial" component. No increase of relative sensitivity, $S$ was apparent.
The most interesting property of IAA, however, was that it caused no deterioration of the retina even under continuous perfusion. This difference from all the other SH-reagents suggested that IAA is unable to affect the phototransduction machinery inside the rod. If so, it offers the possibility of distinguishing direct action on the membrane channels from e.g. phosphodiesterase inhibition. We endeavoured to demonstrate this by giving the isolated rod first a lengthy exposure to IAA, then switching to a perfusate containing both IAA and the phosphodiesterase inhibitor IBMX. We should expect to obtain first pure effects on the sodium channels of the plasma membrane, then (after adding IBMX) mixed (channel plus internal) effects somewhat as seen in the later phases of perfusion with NEM, PHMB or PCMP.

Figure 6 illustrates the outcome of one such experiment. In agreement with the experiments where the other SH-reagents were used, the "membrane" effect involved a transitory increase in current, a growth of the saturating response amplitude, and the appearance of a leakage current which could not be abolished by light. The responses did not slow down significantly, and there was no continuous change in $S$ or $U_{\max }$. The subsequent addition of IBMX first brought about an additional increase in dark current and photoresponses, as is known to be associated with the inhibition of phosphodiesterase, and then set off the characteristic slow effects. Sensitivity started

A

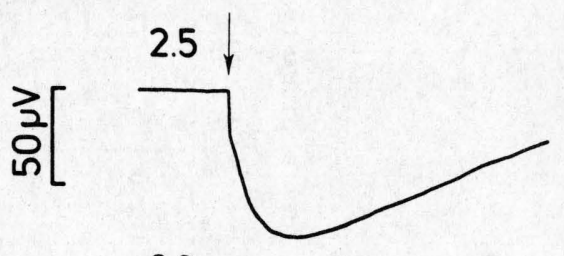

B
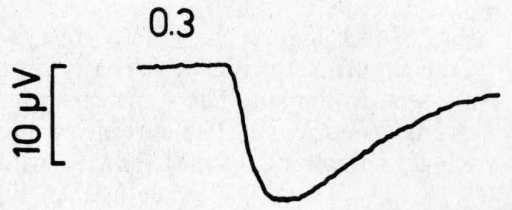

C
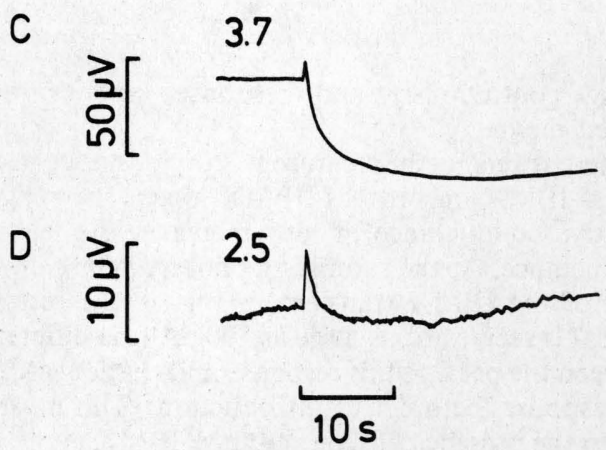

Fig. 4. Biphasic responses observed after long perfusion in $0.5 \mathrm{mM}$ NEM Ringer. The log flash intensities are given above the responses, as in Fig. 1.(A) A saturated response, (B) a response to a dim flash before the application of NEM. (C) and (D) responses to high intensities $25 \mathrm{~min}$ and $42 \mathrm{~min}$ after switching to NEM. Note that the intensity in (D) is the same as in (A), but the voltage scale is five times larger. 

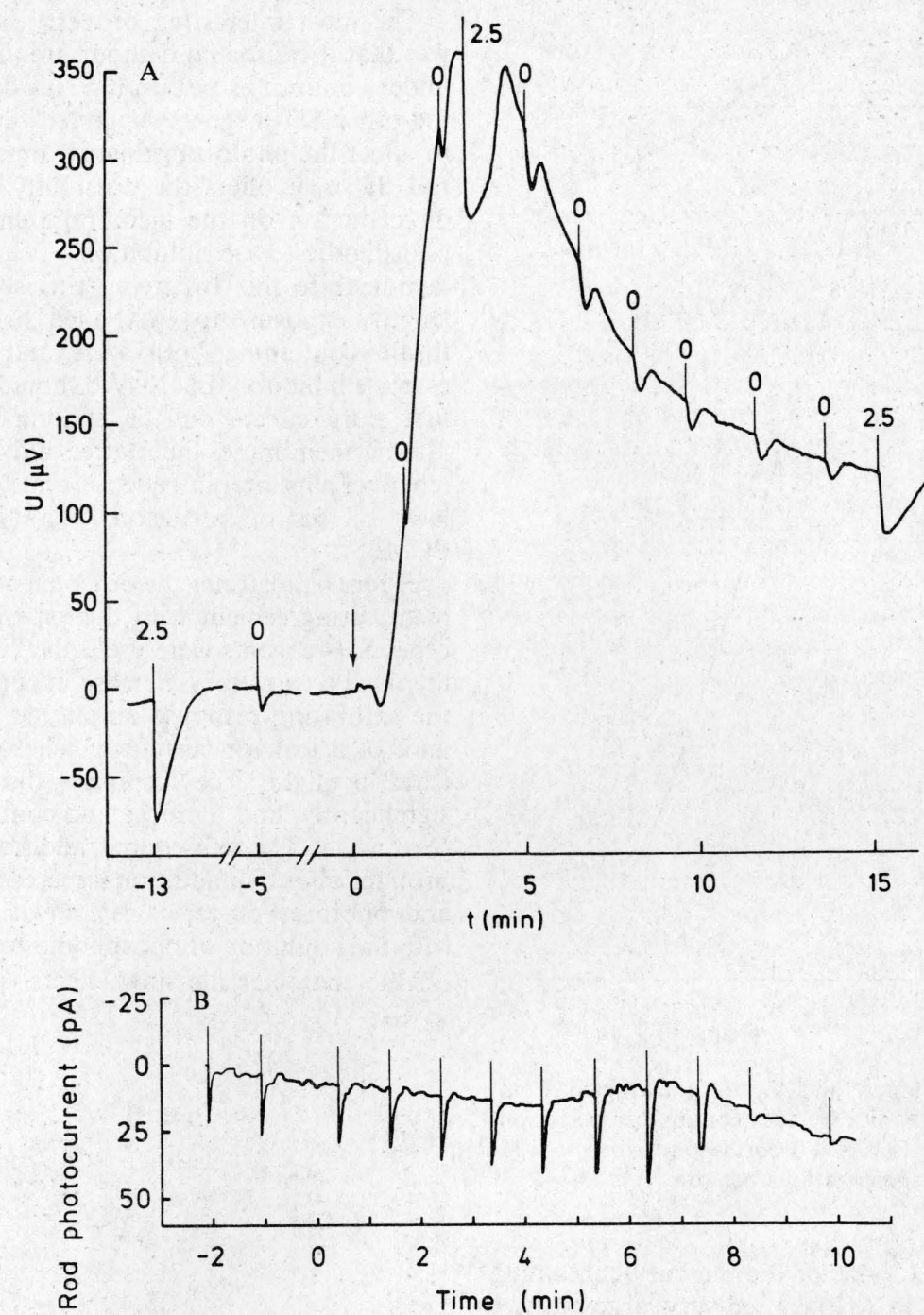

Fig. 5. (A) Transretinal ERG voltage transient brought about by a $0.5 \mathrm{~min}$ pulse of $1 \mathrm{mM}$ PHMB. Conventions as in Fig. 1. A nearly saturated response and a small response before the drug application are seen to the left. The transients seen in PCMP, IAA and DTT were qualitatively similar but varied strongly in size. (B) The current of an isolated rod before and after a switch from standard Ringer to Ringer containing $0.5 \mathrm{mM}$ PHMB. All flashes had the same high intensity ( $\log I=-0.5$ on the relative scale used in Fig. 2B). (Retraced from chart recording.)

falling continuously and response kinetics were slowed down.

There is one further feature worthy of notice in the IAA + IBMX record: as IBMX opens new lightsensitive conductance, it also increases the leakage conductance. At the saturating photoresponse on the crest of the IBMX transient in Fig. 6, the residual current is seen to be as large as $100 \mathrm{pA}$ (the difference between the peak of this response and that of saturating responses before drug introduction). This must be due to the presence of IAA, because IBMX alone has not been found to open any light-insensitive conductance (Cervetto and McNaughton, 1986).

\section{The effects of DTT}

DTT is a reagent which breaks S-S-bridges, thus keeping the SH-groups in the reduced state. Its fast effects on isolated salamander rods were to increase the dark current and the saturated response amplitude correspondingly. Like IAA, it caused no decay of sensitivity or dark current even in continuous perfusion, suggesting that it did not affect the transduction machinery. In the intact retina, there were not discernible effects on photoresponses at all: the only clear effect of DTT on frog ERG was to produce enormous positive "glial" transients, up to $80 U_{0}$ ( $5 \mathrm{mM}$ DTT, continuous perfusion). This suggests that in rods in situ oxidative S-S-bridge formation is comparatively slight.

\section{DISCUSSION}

The initial increase in rod current

All the SH-reagents, as well as DTT, initially increased the dark current of the rods. This could be 


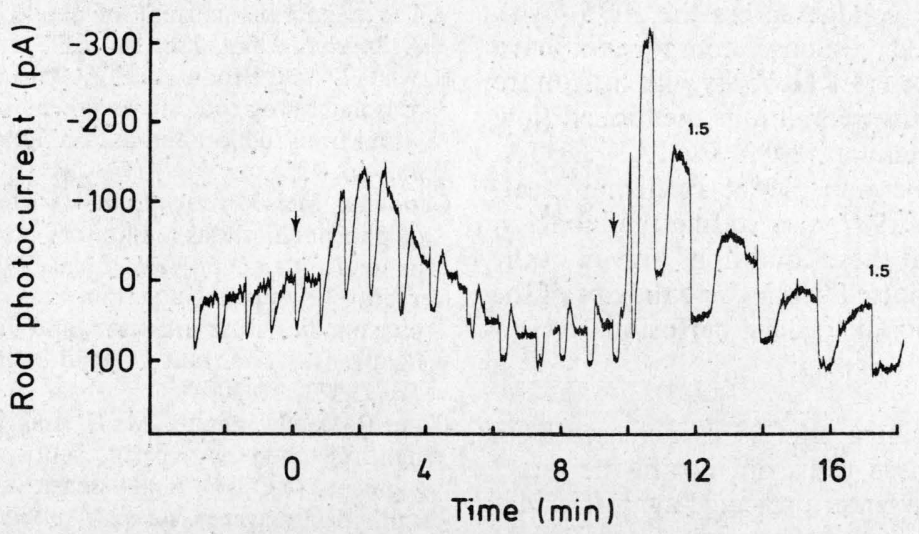

Fig. 6. The current of an isolated rod during the following experimental sequence: (1) standard Ringer, (2) after a switch to Ringer containing 2 mM IAA (first arrow); (3) after a further switch to Ringer containing $2 \mathrm{mM}$ IAA plus $0.2 \mathrm{mM}$ IBMX (second arrow). All flashes had the same high intensity (log $I=0$ on the relative scale used in Fig. 2B), except the two marked $1.5(\log I=1.5)$. All responses are saturated.

due either to direct action on the channels, increasing the conductivity of each channel, or to action on the transduction machinery, increasing the number of open channels via elevated cGMP levels. In the case of NEM, PHMB and PCMP, we have no strong evidence to distinguish between these possibilities, but our experiments with IAA plus IBMX (see Fig. 6) do suggest that SH-reagents exert both types of effects. The presence of a direct channel effect is further suggested by the results with DTT, and is in agreement with the results of Cone et al. (1984). In osmotic studies, these authors found that the restoration of the $\mathrm{Na}^{+}$-permeability to control level, after it had been abolished by oxidizing agents or bright light, required no more than $10^{5}$ molecules of NEM bound to each ROS. With some $10^{7}$ molecules of PDE and some $10^{8}$ G-proteins in each ROS (Pugh and Cobbs, 1986), it is difficult to see how such a small number of molecules could significantly inhibit the enzymatic transduction cascade.

The increase in dark current involved a component which was not sensitive to light: saturating lights no longer brought the rod current down to zero. Although this new leakage component constituted but a small proportion of the total rod current, it could (on the crest of the initial transient) be of the same magnitude as the entire pre-drug dark current.

Any increase in rod current due to increased sodium conductance is by necessity transitory. As the $\mathrm{Na}^{+}$concentration gradient falls, reducing the driving force for that ion, the current will fall back although the large conductance may persist. The steady-state current must, of course, correspond to the sodium pumping rate.

\section{Slow effects}

Loss of sensitivity. In continuous perfusion, all the $\mathrm{SH}$-reagents save IAA gradually destroyed the lightsensitivity of the rod. Sensitivity fell much more steeply than the maximum response amplitude, indicating that the gain of phototransduction was reduced. This, as well as the relative slowness of the process, suggests that the main action was on the enzymatic machinery, requiring that the reagents penetrate into the cell.

Although we have emphasized that the "internal" effects are similar to those found in connection with phosphodiesterase inhibition, the physiological picture is equally consistent with inhibition at other points in the transduction chain. Reichert and Hofmann (1984) observed that the binding of NEM to the SH-groups of the G-protein prevents the binding of the photoisomerized rhodopsin molecule. At present, we cannot localize the internal action of NEM more precisely.

In continuous NEM perfusion, log sensitivity decreased at a near-constant rate (cf. Fig. 3), i.e. sensitivity fell exponentially. This is consistent with first-order reaction kinetics, depleting a sensitivitycontrolling enzyme. Assume that the inactivation rate is proportional to the concentrations of active enzyme and NEM, here denoted $E$ and $c_{0}$ :

$$
\mathrm{d} E / \mathrm{d} t=-k c_{0} E
$$

where $c_{0}$ is constant when cytoplasmic NEM has reached a steady-state value, and $k$ is a rate constant. Then the concentration of active enzyme (not bound to NEM) would decrease exponentially:

$$
E=C \exp \left(-k c_{0} t\right)
$$

where $C$ is a constant. The time constant of the decay $\left(\tau=1 / k c_{0}\right)$ is inversely proportional to $c_{0}$, in qualitative agreement with our experimental results.

The biphasic responses. Earlier, we have demonstrated that biphasic current responses with initial positive humps may be recorded if two conditions are met. First, the recording geometry gives relatively more weight to current entering the ROS near the tip than current entering near the base. Secondly, the sodium channels in the tip are operative but phototransduction there has relatively low sensitivity and/or slow kinetics (cf. Baylor et al., 1979; Schnapf, 1983). The basic idea is that a light flash may then produce a transitory increase in current flowing the longer distance from the inner segment to the outer segment tips, which will under certain conditions give 
a net increase in the voltage across the ROS layer. Indeed, such inverted response components have been observed in various ERG works with little more in common than the two conditions mentioned (low $\mathrm{Ca}^{2+}, \mathrm{CH}_{3} \mathrm{SO}_{3}^{-}$: Greenblatt, 1983; EGTA + EDTA: Govardovskii and Berman, 1985; oxidizing treatments: Donner et al., 1987, also stating the model in detail). It is likely that these conditions prevail in the ERG experiments reported here, where the tips of the rods are directly exposed to drug perfusion.

\section{Conclusions}

The disulfide reducing agent, DTT, increased ROS conductivity in perfused rods, but not in the intact retina, where rods are better protected against oxidation. This suggests that the effect in isolated rods at least partly represents a restoration of "natural" conductance which has been lost because of excessive oxidation of SH-groups.

SH-binding reagents had a powerful effect on rods in the intact retina as well. This indicates that, by binding to SH-groups, they modify the properties of the channel molecule so as to cause a larger-thannormal conductivity. Part of this large conductance is insensitive to light. The subsequent, more dramatic, fall in light sensitivity appears to be due mainly to the inactivation of the enzymatic machinery by drug molecules which have entered the rod. Initially, the inhibition of transduction enzymes would also act to increase conductance (by elevating cGMP levels), contributing part of the large dark current transient seen e.g. with NEM.

In the frog ERG, all the reagents (with the possible exception of NEM) caused further substantial voltage transients that were not associated with changes in rod current. These are probably due to action on Müller cells and need not concern us here, except that the great variability in the amplitude of these glial currents emphasizes the differences between the SHreagents. However, in view of all potential variables (e.g. affinities of binding to different SH-groups and capacities of traversing cell membranes), the broad uniformity of their action appears more telling than the differences.

Acknowledgements - We are grateful for the valuable insights provided by Dr Grigorii Kalamkarov. This work was supported by the Academy of Finland (Grant no. 01/455).

\section{REFERENCES}

Bäckström A.-C. and Hemilä S. O. (1979) Dark-adaptation in frog rods: changes in the stimulus-response function. J. Physiol., Lond. 287, 107-125.

Baylor D. A., Hodgkin A. L. and Lamb T. D. (1974) The electrical response of turtle cones to flashes and steps of light. J. Physiol., Lond. 242, 685-727.

Baylor D. A., Lamb T. D. and Yau K.-W. (1979)
The membrane current of single rod outer segments. J. Physiol., Lond. 288, 589-611.

Bownds D. and Brodie A. E. (1975) Light-sensitive swelling of isolated frog rod outer segments as an in vitro assay for visual transduction and dark adaptation. J. gen. Physiol. 66, 407-425.

Capovilla, M., Cervetto, L. and Torre, V. (1983) The effect of phosphodiesterase inhibitors on the electrical activity of toad rods. J. Physiol., Lond. 343, 277-294.

Cervetto, L. and McNaughton, P. A. (1986). The effects of phosphodiesterase inhibitors and lanthanum ions on the light-sensitive current of toad retinal rods. J. Physiol., Lond. 370, 91-109.

Cone R. A., Usselman, M. C. and Wu D.-Z. (1984) The light-regulated $\mathrm{Na}^{+}$permeability of isolated rod outer segments (ROS) is highly sensitive to oxidation and to sulfhydryl reagents. Invest. Ophthal. Vis. Sci. 25 (suppl.) 287 (abstract 41).

Derevyanchenko T. G., Fedorovich I. B. and Ostrovskii M. A. (1985) Axial distribution of sulfhydryl groups in the outer segments of frog retinal rods. Tsitologiya 27, 1197-1199 (in Russian).

Donner K. and Hemilä S. (1985) Rhodopsin phosphorylation inhibited by adenosine in frog rods: lack of effects on excitation. Comp. Biochem. Physiol. 81A, 431- 439.

Donner K., Hemilä S. and Koskelainen A. (1987) Transient sensitivity reduction and biphasic photoresponses observed when frog retinal rods are oxidized. Comp. Biochem. Physiol. 87A, 749-756.

Donner K., Hemilä S. and Koskelainen A. (1988) Temperature-dependence of rod photoresponses from the aspartate-treated retina of the frog (Rana temporaria). Acta Physiol. Scand. 134, 535-541.

Folkman J. and Moscona A. (1978) Role of cell shape in growth control. Nature, Lond. 273, 345-349.

Govardovskii V. I. and Berman A. L. (1985) Effects of divalent cation chelators on the rod receptor potential of the frog retina. Dokl. Akad. Nauk SSSR 281, 1000-1004 (in Russian).

Hemilä S. (1977) Background adaptation in the rods of the frog's retina. J. Physiol., Lond. 265, 721-741.

Hemilä S. (1983) The mass receptor potential in the study of enzymatic processes in the vertebrate retinal photoreceptors. Acta Polytech. Scand., appl. Phys. 138, 50-57.

Hemilä S. (1987) The stimulus-response function of visual systems. Vision Res. 27, 1253-1261.

Lamb T. D., McNaughton P. and Yau K.-W. (1981) Spatial spread of activation and background desensitization in toad rod outer segments. J. Physiol., Lond. 319, 463-496.

Ostrovskii M. A., Fedorovich I. B. and Dontsov A. E. (1987) Photo-oxidation processes in the eye structures. Protecting function of the lens and screening pigments. Biofizika 23, 896-909 (in Russian).

Pugh E. N. Jr and Cobbs W. H. (1986) Visual transduction in vertebrate rods and cones: a tale of two transmitters, calcium and cyclic GMP. Vision Res. 26, 1613-1643.

Reichert J. and Hofmann K. P. (1984) Sulfhydryl group modification of photoreceptor $G$ protein prevents its light-induced binding to rhodopsin. FEBS Lett. 168, 121-124.

Schnapf J. L. (1983) Dependence of the single photon response on longitudinal position of absorption in toad rod outer segments. J. Physiol., Lond. 343, 147-159.

Wormington C. M. and Cone R. A. (1978) Ionic blockade of the light-regulated sodium channels in isolated rod outer segments. J. gen. Physiol. 71, 657-681. 